\title{
Zooming Towards Better Health: Participant Experience in a Web-Based Group Cognitive Behavioral Intervention
}

\author{
Jannat Saini ${ }^{1,2}$, PharmD; Ashley Owen ${ }^{1}$, PhD; Anna Hammer ${ }^{1}$, LAMFT; William Brown ${ }^{1}$, BS; Ambar Kulshreshtha ${ }^{1}$,
} $\mathrm{MD}, \mathrm{PhD}$

\footnotetext{
${ }^{1}$ Department of Family \& Preventative Medicine, Emory University, Atlanta, GA, United States

${ }^{2}$ Rollins School of Public Health, Emory University, Atlanta, GA, United States
}

\section{Corresponding Author:}

Jannat Saini, PharmD

Department of Family \& Preventative Medicine

Emory University

4500 N Shallowford Road

Atlanta, GA, 30338

United States

Phone: 14129990543

Fax: 14047786920

Email: jannat.saini@emory.edu

\section{Abstract}

Background: Internet-based Cognitive Behavioral Therapy (CBT) is effective in the management of several medical conditions such as anxiety and depression. The CBT method addresses the interaction between people's thoughts, feelings, and behavior, and has also been shown to be an effective strategy for patients with dementia and comorbid anxiety. However, no study has evaluated an internet-based group CBT for prevention of dementia in high risk patient groups such as African American people. African American people have twice the risk of developing dementia compared to white people. Therefore, more targeted and easy-to-access interventions are urgently needed in this group.

Objective: The purpose of this study was to survey participant evaluation of the Web-based CBT program for African American people with mild cognitive impairment.

Methods: The LIGHT (Lifestyle Intervention Guidance for a Healthier Tomorrow) study program is a six-month pilot study that aims to test the feasibility and acceptability of CBT in African American people with a diagnosis of MCI through group video conferencing compared with in-person group CBT sessions. Ten participants were randomized to each study arm. An anonymous online survey using Likert scales was administered to study participants to measure the acceptability and feasibility of CBT using Web-based video conferencing via the Zoom application after each session. The survey also included open-ended questions to gauge additional feedback from the participants.

Results: Eighty-three percent of participants (5 out of 6 respondents) in the Web-based arm rated the ease of use of the Zoom technology positively (ie, either very easy or easy to use on a Likert scale). Sixty-seven percent of participants (4 out of 6 respondents) rated that the computerized CBT can be an effective medium to improve health and healthy behaviors. All respondents rated the helpfulness of the online sessions as "most helpful" (50\%), "helpful" (33\%), or "neutral" (17\%). In response to open-ended questions, participants stated that the online sessions were "on spot, in the moment counseling," provided "the ability to interact with others of varied backgrounds, experiencing similar issues," "convenience of being at home versus traveling to a particular location is a tremendous plus," and that "(the sessions) afford the group a certain level of anonymity with the choice of when and/or whether or not to share in the discussions." Other key phrases that were stated included saving travel expenses, time, being cost efficient but at the same time allowing a diverse population to participate. Participants mentioned having the occasional technical difficulty, and one respondent mentioned the lack of physical interaction as a disadvantage. Four participants did not complete the survey.

Conclusions: African American participants with MCI rated Web-based group CBT interventions as helpful to improve healthy behaviors. Participants noted that that Web sessions are easy to use, as they can interact with each other in a group setting from their own homes. Our survey provides preliminary evidence regarding the potential for a Web-based group CBT protocol as a useful modality for interventions designed to improve lifestyle and reduce the risk of dementia in African American people. Ongoing research will further test the effectiveness of the Web-method of treatment delivery. 
(iproc 2018;4(2):e11773) doi: $10.2196 / 11773$

\section{KEYWORDS}

African American; CBT; cognitive impairment not dementia; computerized cognitive behavior therapy; online survey; online CBT; MCI

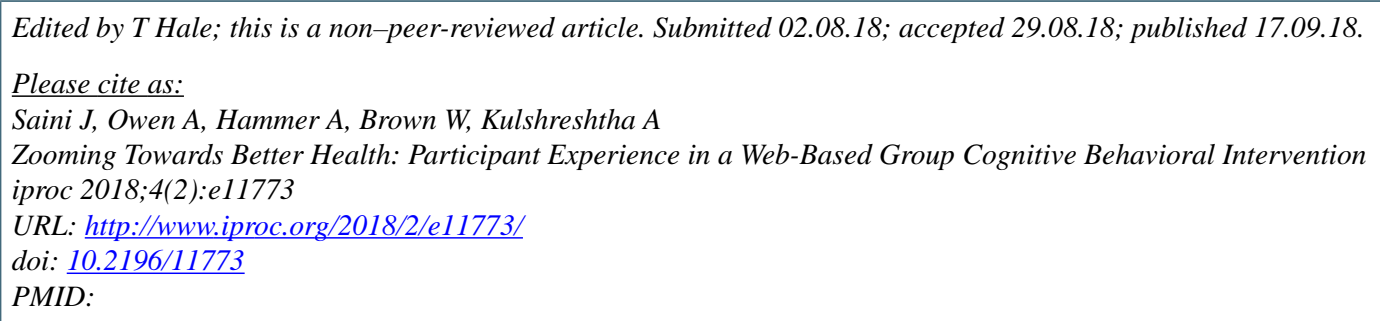

(CJannat Saini, Ashley Owen, Anna Hammer, William Brown, Ambar Kulshreshtha. Originally published in Iproceedings (http://www.iproc.org), 17.09.2018. This is an open-access article distributed under the terms of the Creative Commons Attribution License (https://creativecommons.org/licenses/by/4.0/), which permits unrestricted use, distribution, and reproduction in any medium, provided the original work, first published in Iproceedings, is properly cited. The complete bibliographic information, a link to the original publication on http://www.iproc.org/, as well as this copyright and license information must be included. 